\title{
NEUROBLASTOMA AS A DIFFERENTIAL DIAGNOSIS OF CHRONIC RECURRENT MULTIFOCAL OSTEOMYELITIS (CRMO): CASE REPORT
}

\author{
Rodrigo Maldonado Franco ${ }^{1, \star}$, Ana Caroline Oliveira de Lima Grossi ${ }^{1}$, Bruno Carnevalli ${ }^{1}$, Gustavo Lawin ${ }^{1}$, Milene Tieno Sakata \\ Vasconcellos ${ }^{1}$, Priscila Beatriz de Souza Medeiros ${ }^{1}$
}

1.Universidade Estadual de Londrina, Londrina (PR), Brazil.

*Corresponding author: rmfranco@outlook.com

\section{BACKGROUND}

Chronic recurrent multifocal osteomyelitis (CRMO) is an autoinflammatory bone disease. There are foci of nonbacterial bone inflammation, with pain in the affected sites, which may present with low-grade fever, inflammatory diseases of the skin or gastrointestinal tract, as well as arthritis adjacent to bone lesions. It has an unknown etiology, predominantly in female, children and adolescents. We describe the case of a patient with neuroblastoma, but with clinical manifestations and imaging tests that were initially suggestive of CRMO.

\section{CASE REPORT}

A 7-year-old male child, previously healthy, started 7 months ago with intermittent fever, arthralgia and migratory arthritis in knees, shoulders, elbows and lumbar spine, evolving with progressive worsening of the intensity and frequency of symptoms. In his evaluation, he had arthritis of the right shoulder, left hip, right knee and low back pain, with marked increase in inflammatory tests, but normal radiographs of affected joints. He was first medicated with an anti-inflammatory, with the hypothesis of juvenile idiopathic arthritis, starting treatment with methotrexate and corticosteroids. After initial improvement with therapy, he returned reporting worsening of pain after discontinuation of the anti-inflammatory, and the corticosteroid reduction, with the following exams: magnetic resonance imaging (MRI) of sacroiliac joints: hypervascularized bone lesions in L3, L5, sacral bodies, iliac and acetabular roofs bilaterally, which may be due to an inflammatory/infectious process, including CRMO, similar lesions in the femur bilaterally. Bone scintigraphy: lytic lesion in the 1st lumbar vertebra, areas with increased osteogenic activity in the skullcap, thoracic and lumbar vertebras, sternum, costal arches bilaterally, distal third of humerus, sacroiliac joints, right iliac crest, middle third of the right and left femur-which may correspond to infiltrative processes (secondary bone lesions), suggesting an associated inflammatory process in sacroiliac joints (sacroiliitis)—, whole body MRI, multiple diffuse bone lesions in the axial and peripheral skeleton, lower cervical lymph node enlargement on the left, retroperitoneal lymph node block. In view of the new data, bone and marrow biopsy was indicated, with immunohistochemistry indicating poorly differentiated metastatic neuroblastoma. The patient was then referred to the Pediatric Oncology Service.

\section{CONCLUSION}

In this report, a patient with neuroblastoma had musculoskeletal manifestations and radiological images suggestive of CRMO. This fact reinforces the need to review the diagnosis in the event of an unusual evolution of a rheumatologic condition, including oncohematological diseases in the differential diagnosis.

\section{KEYWORDS}

CRMO, Neuroblastoma, Autoinflammatory, Osteomyelitis. 